\title{
ARTICLE \\ Psychostimulant drug effects on glutamate, Glx, and creatine in the anterior cingulate cortex and subjective response in healthy humans
}

\author{
Tara L. White ${ }^{1,2}$, Mollie A. Monnig ${ }^{1}$, Edward G. Walsh ${ }^{3}$, Adam Z. Nitenson ${ }^{4}$, Ashley D. Harris ${ }^{5}$, Ronald A. Cohen ${ }^{6}$, Eric C. Porges ${ }^{6}$, \\ Adam J. Woods ${ }^{6}$, Damon G. Lamb ${ }^{6,7,8}$, Chelsea A. Boyd ${ }^{9}$ and Sinda Fekir ${ }^{4}$
}

Prescription psychostimulants produce rapid changes in mood, energy, and attention. These drugs are widely used and abused. However, their effects in human neocortex on glutamate and glutamine (pooled as Glx), and key neurometabolites such as $\mathrm{N}$ acetylaspartate (tNAA), creatine (tCr), choline (Cho), and myo-inositol (Ins) are poorly understood. Changes in these compounds could inform the mechanism of action of psychostimulant drugs and their abuse potential in humans. We investigated the acute impact of two FDA-approved psychostimulant drugs on neurometabolites using magnetic resonance spectroscopy ( ${ }^{1} \mathrm{H}$ MRS). Single clinically relevant doses of $d$-amphetamine (AMP, $20 \mathrm{mg}$ oral), methamphetamine (MA, $20 \mathrm{mg}$ oral; Desoxyn ${ }^{\circledR}$ ), or placebo were administered to healthy participants $(n=26)$ on three separate test days in a placebo-controlled, double-blinded, within-subjects crossover design. Each participant experienced all three conditions and thus served as his/her own control. ${ }^{1} \mathrm{H}$ MRS was conducted in the dorsal anterior cingulate cortex (dACC), an integrative neocortical hub, during the peak period of drug responses (140-150 $\mathrm{m}$ post ingestion). $D$-amphetamine increased the level of Glu $(p=.0001)$, Glx $(p=.003)$, and $\operatorname{tCr}(p=.0067)$ in the dACC. Methamphetamine increased Glu in females, producing a significant crossover interaction pattern with gender ( $p=.02)$. Drug effects on Glu, $\mathrm{tCr}$, and Glx were positively correlated with subjective drug responses, predicting both the duration of AMP liking (Glu: $r=+.49, p=.02 ; \mathrm{tCr}: r=+.41, p=.047$ ) and the magnitude of peak drug high to MA (Glu: $r=+.52, p=.016 ; \mathrm{Glx}: r=+.42, p$ $=.049$ ). Neither drug affected the levels of tNAA, Cho, or Ins after correction for multiple comparisons. We conclude that $d$ amphetamine increased the concentration of glutamate, Glx, and $\mathrm{tCr}$ in the dACC in male and female volunteers $2 \frac{1}{2}$ hours after drug consumption. There was evidence that methamphetamine differentially affects dACC Glu levels in women and men. These findings provide the first experimental evidence that specific psychostimulants increase the level of glutamatergic compounds in the human brain, and that glutamatergic changes predict the extent and magnitude of subjective responses to psychostimulants.

Neuropsychopharmacology (2018) 43:1498-1509; https://doi.org/10.1038/s41386-018-0027-7

\section{INTRODUCTION}

Psychostimulant drugs are widely used and often misused by young adults [1]. Prescription psychostimulants first act by blocking and reversing monoamine transporters, producing a supranormal increase in dopamine (DA), norepinephrine (NE), and serotonin $(5-\mathrm{HT})[2,3]$. Monoamines play a role in tuning the signal to noise ratio of incoming stimuli (NE), facilitating the speed and flexibility of switching between competing cues (DA), and setting activational thresholds for stimulus detection and response (5-HT) [4-9]. These neuromodulatory effects involve other neurotransmitter systems, of which glutamate (Glu) is the most ubiquitous. Glu mediates the vast majority of excitatory neurotransmission and has a fundamental role in learning, memory, and retention of learned cues and events [10-12]. Despite this critical role, little is known about the acute glutamatergic impact of psychostimulant drugs in humans. Insight about these effects is essential for understanding the immediate mechanism of action of psychostimulant drugs and their abuse potential in vulnerable individuals.

Several lines of evidence indicate that psychostimulant drugs are likely to acutely impact Glu and key neurometabolites in human neocortex. Chronic users of stimulant drugs show elevated levels of Glu, myo-inositol (Ins), and choline (Cho), and reduced levels of $\mathrm{N}$-acetylaspartate (tNAA) and creatine ( $\mathrm{tCr}$ ) compared to non-drug users in a variety of cortical regions [13-21]. These findings suggest long-term drug-related changes in excitatory neurotransmission (Glu), intracellular catecholamine signaling (Ins), cell membrane turnover and production of acetylcholine (Cho), neuronal integrity (tNAA), and bioenergetics (tCr), respectively [22]. Glx, a composite measure of Glu and glutamine (Gln) $[22,23]$, shows biphasic changes during stimulant drug abstinence

\footnotetext{
${ }^{1}$ Center for Alcohol and Addiction Studies, Brown University, Providence, RI, USA; ${ }^{2}$ Department of Behavioral and Social Sciences, School of Public Health, and Institute for Brain

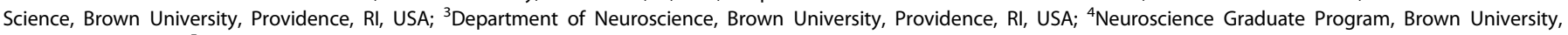

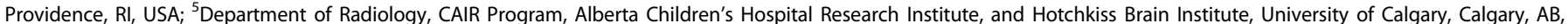

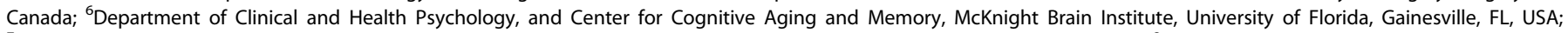

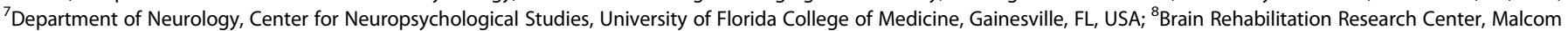
Randall Veterans Affairs Medical Center, Gainesville, FL, USA and ${ }^{9}$ Neuroscience Undergraduate Program, Brown University, Providence, RI, USA Correspondence: Tara L. White (Tara_White@Brown.edu)
} 
and recovery from stimulant drug dependence [13]. Further, levels of Glx, Glu, tNAA, and Cho change alongside phasic drug-induced and experiential changes in emotion and alertness in adults without chronic drug exposure. For instance, in healthy adult volunteers, a single intravenous dose of the anxiogenic drug CCK4 induces panic and increases Glx 2-10 min after drug infusion [24]. Low-dose ketamine, a Glu NMDA-receptor antagonist used clinically as a fast-acting antidepressant, causes a phasic increase in Glu and Gln in the anterior cingulate in healthy adults [25]. Anesthetic drugs also produce acute reductions in Glu, Glx, tNAA, and Cho that coincide with drug effects on sedation [26]. Glx appears particularly responsive, and rises following individual epileptic seizure events [27], transcranial magnetic stimulation events, and transcranial direct current stimulation [28]. Work in vivo and in vitro also demonstrate both acute and chronic psychostimulant drug effects on Glu synthesis, catabolism, transport, and release [29-32], indicating multiple points of action on the glutamatergic metabolic cascade. Information about acute drug effects on Glx and Glu is thus necessary to understand the immediate downstream mechanisms of psychostimulant drugs in healthy young adults, and between-person factors that may contribute to stimulant use and misuse in this population.

$d$-Amphetamine (AMP) and methamphetamine (MA) are psychostimulants that are commonly prescribed for use in the medical management of attention deficit hyperactivity disorder (ADHD), obesity, and narcolepsy. Young adults also commonly misuse these drugs for non-medical purposes including recreation and cognitive enhancement. The present study used proton magnetic resonance ( ${ }^{1} \mathrm{H}$ MRS) spectroscopic imaging to investigate the acute impact of AMP and MA on Glx and other key neurometabolites in the neocortex of healthy young adults using a within-subject, doubleblinded, placebo (PBO)-controlled crossover design. ${ }^{1} \mathrm{H}$ MRS data acquisition focused on the dorsal anterior cingulate (dACC), a neocortical hub that connects the default mode, frontoparietal control, cingulo-opercular, and salience networks, which mediate psychostimulant drug effects on emotion, salience, and attention [33-35]. We expected AMP and MA to acutely increase levels of Glx, Glu, and Ins within the dACC compared to PBO. These predictions are based upon the psychostimulant drug effects on Glu in animals $[36,37]$, the concordance between Glx and Glu in ${ }^{1} \mathrm{H}$ MRS [23], and the involvement of Ins in the catecholamine secondary messenger cascade $[38,39]$.

\section{METHODS AND MATERIALS}

Study design

$20 \mathrm{mg} d$-amphetamine sulfate (AMP), $20 \mathrm{mg}$ methamphetamine hydrochloride $\left(\mathrm{MA}\right.$; Desoxyn $\left.{ }^{\circledR}\right)$, and placebo (PBO) were investigated in a counterbalanced, double blind, within-subjects crossover design.

Recruitment

Eligible participants received a physical exam and electrocardiogram. Exclusion and inclusion criteria were similar to previous studies $[40,41]$ and excluded those younger than 18 or older than
35; those with BMI below 18 or over 30; left-handedness; less than a high-school education/GED; lack of fluency in English; chronic medical conditions; abnormal EKG; current or past Axis I psychiatric disorder; smoking more than five cigarettes per week; and in females, current or intended pregnancy or lactation. The study was approved by the Institution Review Board for research with human subjects at Brown University and the Memorial Hospital of Rhode Island in accordance with the Code of Federal Regulations (Title 45, Part 46) "Protection of Human Subjects" adopted by the National Institutes of Health and the Office for Protection from Research Risks. The study was conducted ethically in accordance with the Helsinki Declaration of 1964 (revised 2013) and the National Advisory Council on Drug Abuse Recommended Guidelines for the Administration of Drugs to Human Subjects. Written informed consent was obtained from all participants.

\section{Participants}

Twenty-six healthy right-handed individuals participated. Participants were $18-28$ years of age (mean $=22.3$ years, $S D=3.27$ ). The sample was rigorously balanced by gender (13 female, 13 male). Participants were of normal body weight (mean $\mathrm{BMI}=22.3, \mathrm{SD}=$ 1.86; mean body weight $=145.1, \mathrm{SD}=17.4 \mathrm{lb}$ ), and weight did not differ in male and female participants (male mean (SD) $=150.9$ (16.6); female mean (SD) $=139.4$ (16.9); $t(24)=1.74, p=.10$, n.s.). Racial composition was 73\% White, 19\% Asian, 4\% African American, and $4 \%$ Multiracial/other. Ethnicity was $77 \%$ non-Hispanic and $23 \%$ Latino/Hispanic. The sample was well educated, with $8 \%$ reporting a high-school diploma, the majority (61\%) reporting some college education and $31 \%$ reporting a Bachelor's degree or more.

\begin{abstract}
Procedures
The study consisted of seven sessions on separate days: psychological screening, medical screening, orientation, three MRI test sessions, and an exit session. Experimental design appears in Fig. 1. Each test session (AMP, MA, and PBO) was 5.5 $\mathrm{h}$ in duration, conducted at a fixed time of day for each participant to control for circadian effects. Sessions were approximately a week apart (mean interval $9( \pm 8)$ days), with AMP and MA at least 3 days apart to avoid sensitization. For blinding purposes, participants were told that the study capsules could contain one of several classes of drugs (stimulants/appetite suppressants, sedatives/tranquilizers, anti-depressants, and placebo). Participants consumed a standardized meal $2 \mathrm{~h}$ prior to the sessions. Breath alcohol level and urine toxicology screening confirmed participants were drug and alcohol free. Cardiovascular status was evaluated using an Invivo Precess MRI Patient Monitoring System (Soma Technology, Bloomfield, CT) within the scanner and an Omron wrist blood pressure monitor (Omron, Lake Forest, IL) outside the scanner (Fig. 1). Participants were in verbal contact with the experimenter at all times during the experiment.
\end{abstract}

\section{Monetary compensation}

Participants were paid $\$ 220$ for completing the study. Prorated payment was provided to those not completing all three MRI test sessions (\$70/session).

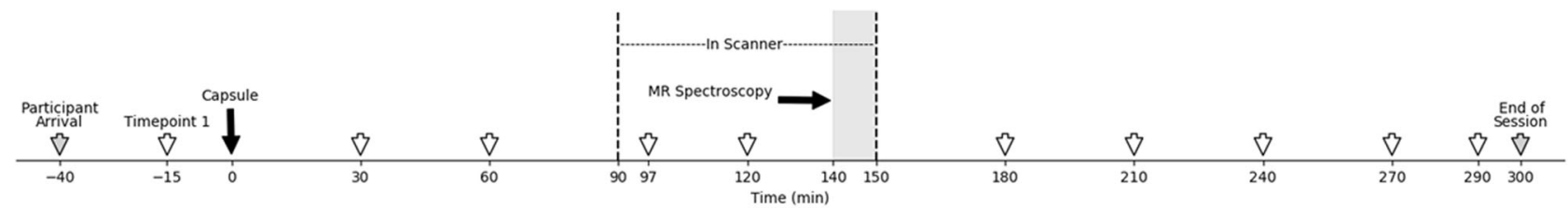

Fig. 1 Experimental design. Timing of MRI test sessions on days 4-6. Sessions were $5.5 \mathrm{~h}$ in duration ( $340 \mathrm{~min}$ total). $X$-axis denotes time relative to administration of the blinded study capsule at time 0 (black arrow). Participants entered the scanner 90 min after administration of the study capsule (vertical dashed black line). MR spectroscopy was conducted 140-150 min post-capsule (gray shading). Physiological data were collected at half hour intervals for internal validity (open arrows). Mood data were collected at half hour intervals outside the scanner to assess subjective drug effects (see Materials and methods for details). Participant arrival and departure times are indicated (gray arrows) 


\section{Study drugs and dosing}

Study drugs were $d$-amphetamine sulfate (AMP, $20 \mathrm{mg}$ oral), methamphetamine hydrochloride (MA, 20 mg oral; Desoxyn ${ }^{\circledR}$ ), and placebo (PBO). Rationale. Dopamine and norepinephrine transporters are sensitive to AMP $[42,43]$. MA differs from AMP in its greater impact at the serotonin transporter [2]. Dose. A 20 $\mathrm{mg}$ oral dose was used for both drugs. This dose is within the range of doses recommended for ADHD, reliably increases subjective measures of stimulant effects $[44,45]$, is equipotent across AMP and MA [46], and is well studied in healthy volunteers [47, 48]. Encapsulation. AMP and MA were individually compounded with dextrose filler and placed in separate opaque, colored gelatin capsules. PBO capsules contained only dextrose. Order. Order of administration was randomized and counterbalanced, with six permutations of drug order across participants (PBO-AMP-MA; PBO-MA-AMP; AMP-PBO-MA; AMPMA-PBO; MA-PBO-AMP; and MA-AMP-PBO). Timing. MRS was conducted 140-150 min after administration of the capsules on each test session (see Fig. 1).

\section{Structural imaging}

A Siemens 3T TIM Trio system (Siemens Medical Solutions, New York, NY) was used for MR data collection. Whole-brain $\mathrm{T}_{1}$ weighted images (MPRAGE sequence) were acquired in the sagittal plane at the beginning of each MRI session (resolution $=1 \mathrm{~mm} \times 1 \mathrm{~mm} \times 1 \mathrm{~mm}$; TR $=1900 \mathrm{~ms}$; TI $=900 \mathrm{~ms}$, TE $=2.98 \mathrm{~ms}$; flip angle $\left.=9^{\circ} ; F O V=256 \times 256\right)$. The structural $T_{1}$ images were processed using SPM12 [49] and Gannet Coregister was used to reconstruct the voxel $[50,51]$.

\section{Spectroscopy}

Spectra were acquired in the dACC using single voxel, pointresolved spectroscopy (PRESS). Voxel size was $15 \times 15 \times 10 \mathrm{~mm}$ $\left(10 \mathrm{~mm}\right.$ in $\mathrm{SI}$ direction) and was placed on the $\mathrm{T}_{1}$-weighted anatomical image using the three-plane reconstructions. The voxel was placed on the dACC immediately anterior and parallel to the corpus callosum (see Fig. 2a). On sagittal view, the midline slice where the corpus callosum was most distinct was selected and the voxel was placed anterior and superior to the corpus callosum. The voxel was rotated to be tangential to the corpus callosum. In coronal, sagittal, and axial views, the voxel was aligned anterior and parallel to the corpus callosum such that the posterior edge of the voxel was in line with the horns of the lateral ventricles. In coronal view, the voxel was right lateralized to capture connectivity with the salience network [52] and minimize CSF contamination from the longitudinal fissure. Voxel placement was confirmed by post hoc voxel reconstruction in subject-specific anatomical space. Reconstructed MRS voxels were checked to confirm agreement with voxel placement protocol for individual anatomy, using subject-specific anatomical landmarks (above). During the MRS sessions, standard first-order auto shimming was implemented, line width checked and shimming manually adjusted to minimize the free water line width. Prior to acquisition of spectral data, non-water-suppressed data sets were obtained to provide for eddy current compensation and to provide a water reference. Repetition time (TR) was $3000 \mathrm{~ms}$, echo time (TE) was $30 \mathrm{~ms}$, with 128 averages taken for a total acquisition time of $6 \mathrm{~m}$ $24 \mathrm{~s}$ for water-suppressed data. The TR of $3000 \mathrm{~ms}$ reduced differential $T_{1}$ effects to less than $12 \%$ and the $T_{2}$ differentiation was minimized by the echo time of $30 \mathrm{~ms}$ [53]. Spectra were processed and metabolites quantified using LCModel [54]. All of the data received zero- and first-order phase correction as part of the regular LCModel process, done automatically by LCModel with no user intervention. Data were compensated for differential $T_{1}$ effects according to individual metabolite $T_{1}$ values at 3 Tesla [53, $55,56]$. Only metabolites with Cramer-Rao lower bound (CRLB) less than or equal to $20 \%$ were evaluated. Example spectra using this procedure are in Fig. $2 b$.

\section{${ }^{1} \mathrm{H}$ MRS measures}

Primary measures. Metabolites reliably assessed by ${ }^{1} \mathrm{H}$ MRS include Glx, a composite measure of Glu and Gln primarily comprised of Glu [23]; tNAA, a combined measure of $\mathrm{N}$-acetyl-aspartate and $\mathrm{N}$ acetylaspartyl-glutamic acid; $\mathrm{tCr}$, a combined measure of creatine and phosphocreatine; Cho, a measure of choline-containing compounds glycerophosphocholine and phosphocholine (GPC + $\mathrm{PCh}$ ); and Ins, a measure of myo-inositol. Reliability for these metabolites is well established $[54,57,58]$. All metabolites were quantified relative to water in institutional units (i.u.) per standard practice [59]. Ratio-based measures were considered but decided against, to avoid interpretive confounds should metabolites change simultaneously, see refs. $[22,60,61]$. Metabolites were corrected for partial volume effects using the formula $[* 1 /(1-\mathrm{fCSF})]$, where fCSF is the voxel volume fraction of cerebrospinal fluid therefore providing a measure of metabolite concentration in tissue within the voxel (per methods of refs. [62-64]). Voxel placement reliability was assessed using dice coefficients $[65,66]$. Subcomponent measures of Glx. Glu and Gln were evaluated separately to provide information on the specificity of drug findings with Glx. LCModel analyzes Glu and Gln using the entire in vivo spectrum in relation to the complete model spectra produced by metabolites at known concentrations in vitro, and provides good discrimination between Glu and Gln when FWHM are on the order of 0.05 ppm [54]. The acquired spectra were uniformly well resolved with an average FWHM of $0.06 \mathrm{ppm}$ and a modal FWHM of $0.05 \mathrm{ppm}$. Mean $( \pm \mathrm{SD})$ CRLB uncertainty was $5( \pm 1) \%$ for Glx, 5( \pm 1$) \%$ for Glu, and $13( \pm 2) \%$ for Gln.

\section{Mood responses}

Subjective responses were evaluated using self-reports on the Drug Effects Questionnaire (DEQ) [67]. Participants indicated on 100-mm lines their response to DEQ items "I LIKE the effects I am feeling right now," rated from "dislike" to "like very much" (DEQ "Like Drug"), and "I am HIGH," rated from "not at all" to "very much" (DEQ "Feel High"). Responses were assessed at half hour intervals outside the scanner, for eight time points of assessment on each session (details in Fig. 1).

\section{Quality control procedures}

Quality control was implemented in three steps: (a) All MRS spectra were visually inspected for quality by an MRS data acquisition and analysis expert (co-author Woods). (b) Using co-registration tools from the Gannet analysis package [51], voxels were reconstructed in each anatomical scan and segmented using SPM12 [50] to allow for quantification of the proportion of white matter, gray matter, and CSF in the voxel. Data were corrected for partial volume effects using the formula $\left[{ }^{*} 1 /(1-\mathrm{fCSF})\right]$, per established methods [62-64]. (c) Data for individual metabolites were excluded, where the CRLB exceeded $20 \%$ SD. These procedures indicated that the majority of the data were of high quality: from a total of 73 acquired scans in 26 participants, 7 scans were excluded for lack of water reference files, and CRLB(Gln) cutoffs were exceeded in 3 instances. Thus, 66 scans in 24 participants were available for analysis, with additional exclusions for $\mathrm{Gln}$ (63 scans).

\section{Statistical analysis}

Drug effects. Linear mixed models with random intercepts were used to evaluate drug effects on each CSF-corrected metabolite individually. Separate analyses examined differences between placebo and AMP, placebo and MA, and AMP and MA. Beta coefficients indicate the model-predicted difference in the metabolite in the active drug condition compared to PBO, with positive beta coefficients indicating higher metabolite levels under drug. Linear mixed models were chosen because the estimation method (i.e., full maximum likelihood estimation) is able to handle missing data without excluding cases listwise, thus making full use of the available data. Effect sizes were calculated using Cohen's $d$ for repeated measures $\left(d_{\mathrm{RM}}\right)$ for within-subjects 
A.

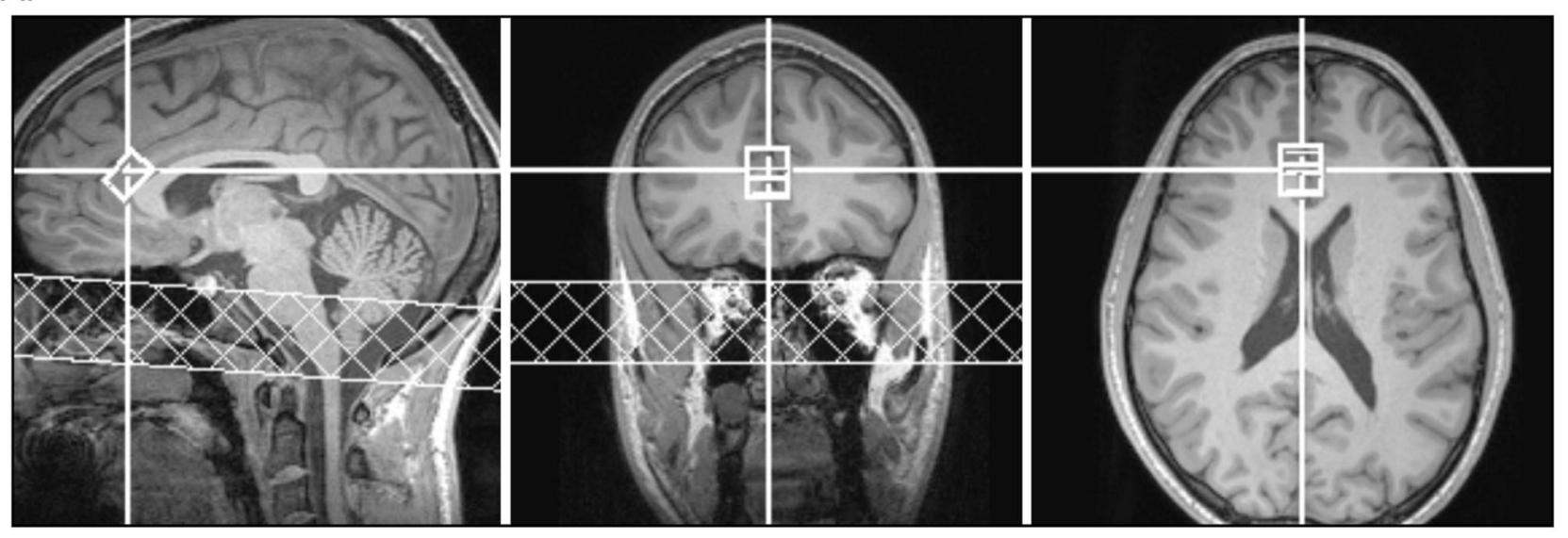

B.

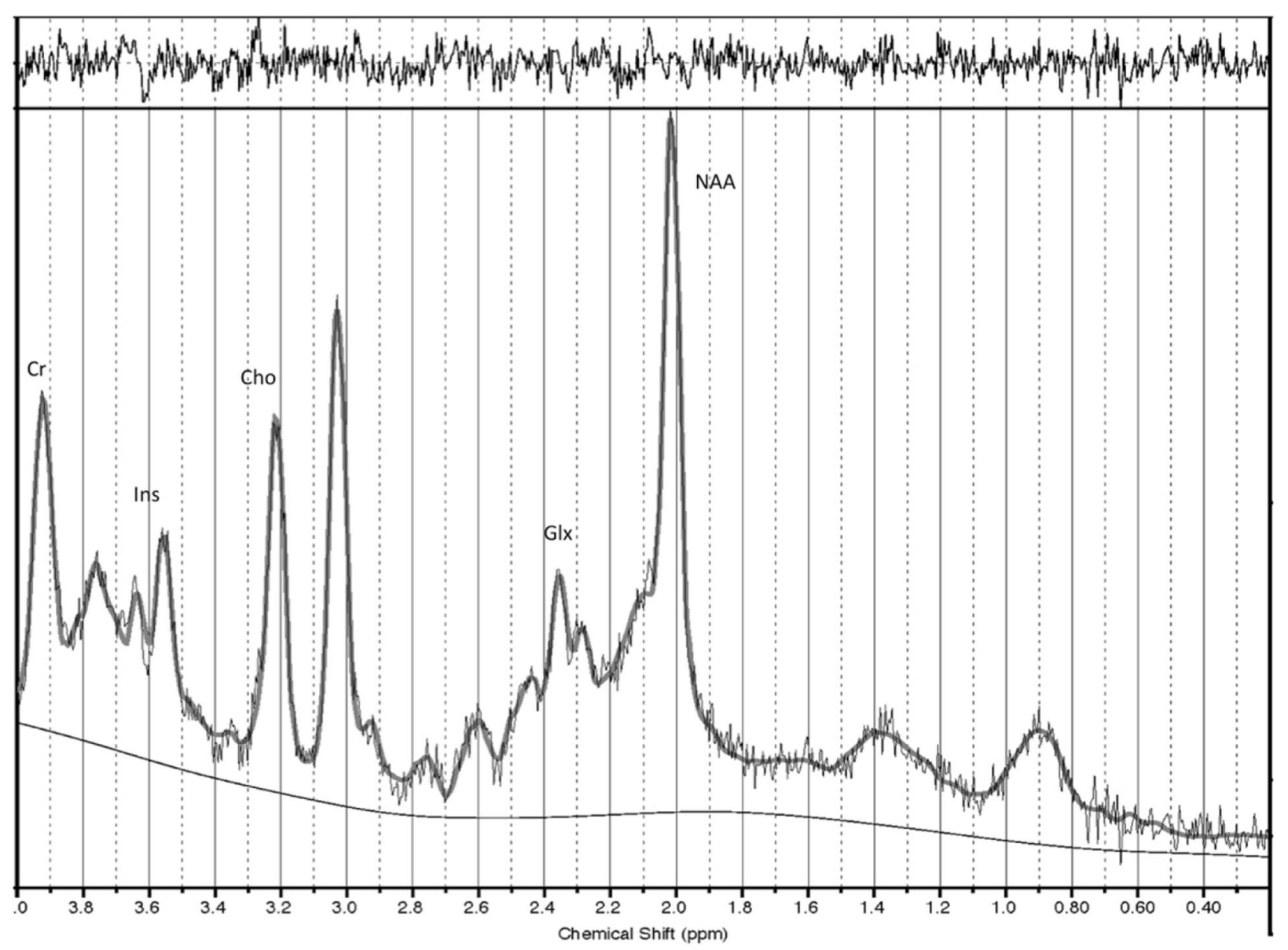

Fig. 2 MRS Voxel and Example Spectra. a Voxel placement in dorsal anterior cingulate cortex (dACC). Left: sagittal; middle: coronal; right: axial views, respectively. b Example MRS spectra with labeled peaks. The solid dark gray curve overlay is the fitted spectrum from LCModel and the raw data shown in light gray, with the baseline below. The upper panel is a plot of the residuals, which in the case of a proper fit appears as noise. Labeled peaks: $\mathrm{Cr}$ creatine, Ins myo-inositol, Cho choline, Glx glutamate and glutamine, NAA N-acetyl-aspartate

effects [68]. Bonferroni correction for seven tests of drug effects on Glx, Glu, Ins, Cho, tCr, tNAA, and Gln was conducted to control family-wise Type I error rate at $a=.05$ (adjusted $a=.0071$ ). Manipulation checks. Sympathomimetic drug activity across the protocol, effectiveness of counterbalancing, reliability of voxel placement across test sessions, \%voxel overlap, structural composition within the voxel, LCModel fit characteristics, gender, and order effects on metabolites were evaluated using repeated measures drug $\times$ time ANOVA models, Pearson $x^{2}$ tests, paired $t$-tests, linear mixed-effects models, and generalized estimation equation (GEE) models (details in Supplementary Methods). Relationship of Glx, Glu, and Gln. The relationship between Glx, Glu, and GIn was assessed using bivariate correlations. Mood correlates. Time-series mood data were reduced to a single metric in each participant, with Time-to-Peak "Like Drug" ratings to AMP and Peak "Feel High" ratings to MA meeting quality control criteria for 
analysis (details in Suppl. Methods). Delta $(\Delta)$ scores quantified drug-induced changes in mood and metabolites relative to $P B O$ in each participant (Mood: $\triangle$ Time-to-Peak DEQ "Like Drug"[AMPPBO]; $\triangle$ Peak DEQ "Feel High"[MA-PBO]; Metabolites: $\triangle$ Glu[AMPPBO], $\triangle$ Glx[AMP-PBO], $\triangle \mathrm{tCr}[\mathrm{AMP}-\mathrm{PBO}] ; \Delta \mathrm{Glu}[\mathrm{MA}-\mathrm{PBO}], \Delta \mathrm{Glx}[\mathrm{MA}-$ $\mathrm{PBO}])$. Positive $\Delta$ scores indicate a longer duration of peak drug liking on AMP compared to PBO ( $\triangle$ Time-to-Peak DEQ "Like Drug"[AMP-PBO]) and a larger drug high on MA compared to PBO $(\triangle$ Peak DEQ "Feel High"[MA-PBO]), and negative scores indicate the inverse. Relationships between drug effects on mood and drug effects on metabolites were evaluated using bivariate correlation. There were three tests of AMP effects ( $\triangle$ Glu[AMPPBO], $\triangle \mathrm{Glx}[\mathrm{AMP}-\mathrm{PBO}], \Delta \mathrm{tCr}[\mathrm{AMP}-\mathrm{PBO}$ ] with $\triangle$ Time-to-Peak DEQ "Like Drug"[AMP-PBO]) and two tests of MA effects ( $\triangle$ Glu[MAPBO], $\triangle \mathrm{Glx}[\mathrm{MA}-\mathrm{PBO}]$ with $\triangle$ Peak DEQ "Feel High"[MA-PBO]). Effect magnitude was estimated by $R^{2}$ and reliability-corrected $R^{2}$ (details in Suppl. Methods). Statistical power. Power analyses for within-subjects effects were conducted in $\mathrm{G}^{*}$ Power 3.1 using an alpha of $.05[69,70]$. The final sample of 24 had high power $(1-\beta$ $=.96)$ to detect large effects $(d=.80)$, adequate power $(1-\beta$ $=.80)$ to detect medium effects $(d \geq .60)$, and low power $(1-\beta$ $=.16)$ to detect small effects $(d=.20)$.

\section{RESULTS}

Internal validity

There was substantial evidence of internal validity, with significant AMP and MA effects on all sympathomimetic outcomes; see Table 1 and Fig. 3 (means in Suppl. Data, Table S1). Sympathomimetic effects of AMP and MA were similar in timing and extent, and began approximately $90 \mathrm{~min}$ post-capsule, peaked $210 \mathrm{~min}$ post-capsule, and remained significant to the end of testing (290 min). Drug effects and drug by time interactions all were significant $(p<.01-.0001$; see Table 1$)$. There was no main effect of gender $(p>.15$, n.s.) and drug by gender interactions were not significant $\left(p>.29\right.$, n.s.). Diastolic blood pressure $\left(\mathrm{BP}_{\mathrm{D}}\right)$, systolic blood pressure $\left(B_{S}\right)$, and heart rate $(\mathrm{HR})$ responses to AMP and $M A$ thus did not differ in males and females. These findings indicate that AMP and MA were effective as dosed, and provide confirmatory evidence of significant drug effects during MRS imaging.

Drug effects on metabolites

Descriptive data and findings for CSF-corrected metabolite concentrations are in Table 2.

\section{Amphetamine (AMP) effect}

There was a main effect of AMP on Glx in the dACC $(B(S E)=1.05$ (.32), $p=.003$; Table 2). The AMP effect on Glx is illustrated in Fig. 4a. The positive beta value indicates that Glx concentrations were higher on AMP than PBO. In terms of magnitude of change, AMP caused a $9 \%$ increase in Glx within the voxel (mean $( \pm S D)=$
$9 \%( \pm 12 \%))$, a medium-sized effect $\left(d_{\mathrm{RM}}=.68\right)$. There was a main effect of AMP on $\mathrm{tCr}(B(S E)=.32(.11), p=.0067)$. In terms of magnitude of change, AMP caused a $5 \%$ increase in $\mathrm{tCr}$ within the voxel (mean $( \pm S D)=5 \%( \pm 8 \%))$, a medium-sized effect $\left(d_{\mathrm{RM}}\right.$ $=.47)$. There was a main effect of AMP on Ins $(B(S E)=.33(.13), p$ $=.022)$, and Cho $(B(S E)=.11(.05), p=.022)$, which were mediumsized effects ( $d_{\mathrm{RM}}=.61$ and .58 , respectively) and did not survive correction for multiple comparisons (see below). There was no effect of AMP on tNAA ( $p>.40)$. Glx subcomponents analysis. Our follow-up analysis showed that Glu concentrations in the dACC were higher on AMP than PBO $(B(S E)=.71(.15), p=.0001)$. The AMP effect on Glu is illustrated in Fig. 4b. In terms of magnitude of change, AMP was associated with an $8 \%$ increase in Glu within the voxel (mean $( \pm S D)=8 \%( \pm 8 \%))$, a medium-sized effect $\left(d_{\mathrm{RM}}=.74\right)$. Gln concentrations did not differ between AMP and PBO $(p>.40)$. Bonferroni correction. The AMP effect on Glu $(p=.0001)$, Glx ( $p$ $=.003)$, and $\mathrm{tCr}(p=.0067)$ survived Bonferroni correction for seven tests (adjusted $a=.0071$ ), a stringent criterion. Interactions. There was no AMP $\times$ gender interaction for Glu $(p=.34$, n.s.; Fig. 5), $\operatorname{Glx}(p=.08, \mathrm{n} . \mathrm{s}$ ), $\mathrm{tCr}(p=.29)$, or any other metabolite ( $p$ 's $>$.19). Pairwise comparisons indicated Glx was higher for females on AMP compared to PBO $(t(20.901)=3.846, \quad p \leq .001$; see Figure S1).

Methamphetamine (MA) effect

There was no main effect of MA on any metabolite $\left(p^{\prime} s>.47\right)$. Heterogeneity. There was between-subject heterogeneity in individual-level Glu responses to MA (Fig. 5). The MA by gender interaction for Glu was significant $(p=.020)$. Relative to PBO, MA significantly increased Glu in females $(t(21.97)=2.333, p=.029)$ and non-significantly decreased Glu in males $(t(22.92)=-1.234, p$ $=.230$ ) (Fig. 5a, b). The gender difference in the linear slope was significant $(B(S E)=-.99(.40), p=.020)$. There was no difference in Glu between men and women on PBO $(t(38.50)=-.484, p=.631)$. In the MA condition, Glu was significantly higher in women compared to men $(t(39.31)=-2.162, p=.037)$. The MA by gender interaction on Glx was not significant $(p>.15$, n.s.). Glx was higher in females after MA than PBO (see Supplemental Figure S1), consistent with the pattern observed for Glu. The MA by gender interaction was not significant for any other metabolite ( $\left.p^{\prime} s>.55\right)$.

\section{Gender}

There were no significant main effects of gender on any metabolite $(p>.11)$.

Manipulation checks Counterbalancing. AMP, MA, and PBO were equally distributed across the three test sessions (Pearson $X^{2}$ test $(1,4)=1.36, p=.85$, n.s.), indicating that drug and order were fully counterbalanced. LCModel fit. LCModel fit characteristics (FWHM, CRLBs [Glu, Gln, Glx], SNR) are presented in Suppl. Data, Table S2. CRLBs, FWHM, and SNR did not differ across drug conditions (CRLB [Glx]: Wald $\chi^{2}(2)=1.339, p=.51$; CRLB[Glu]: Wald $\chi^{2}(2)=3.471, p$

Table 1. Sympathomimetic effects

\begin{tabular}{|c|c|c|c|c|c|c|}
\hline & \multicolumn{2}{|l|}{ AMP } & & \multicolumn{2}{|l|}{ MA } & \\
\hline $\mathrm{BP}_{\mathrm{S}}$ & $25.6^{* * * * * *}$ & $6.17^{* * * * * *}$ & $\mathrm{AMP}>\mathrm{PBO}$ & $44.5^{* * * * * *}$ & $5.69^{* * * * *}$ & $\mathrm{MA}>\mathrm{PBO}$ \\
\hline $\mathrm{HR}$ & $9.11 * *$ & $3.63^{* * * * *}$ & $\mathrm{AMP}>\mathrm{PBO}$ & $36.7^{* * * * * *}$ & $6.87^{* * * * * *}$ & $\mathrm{MA}>\mathrm{PBO}$ \\
\hline
\end{tabular}




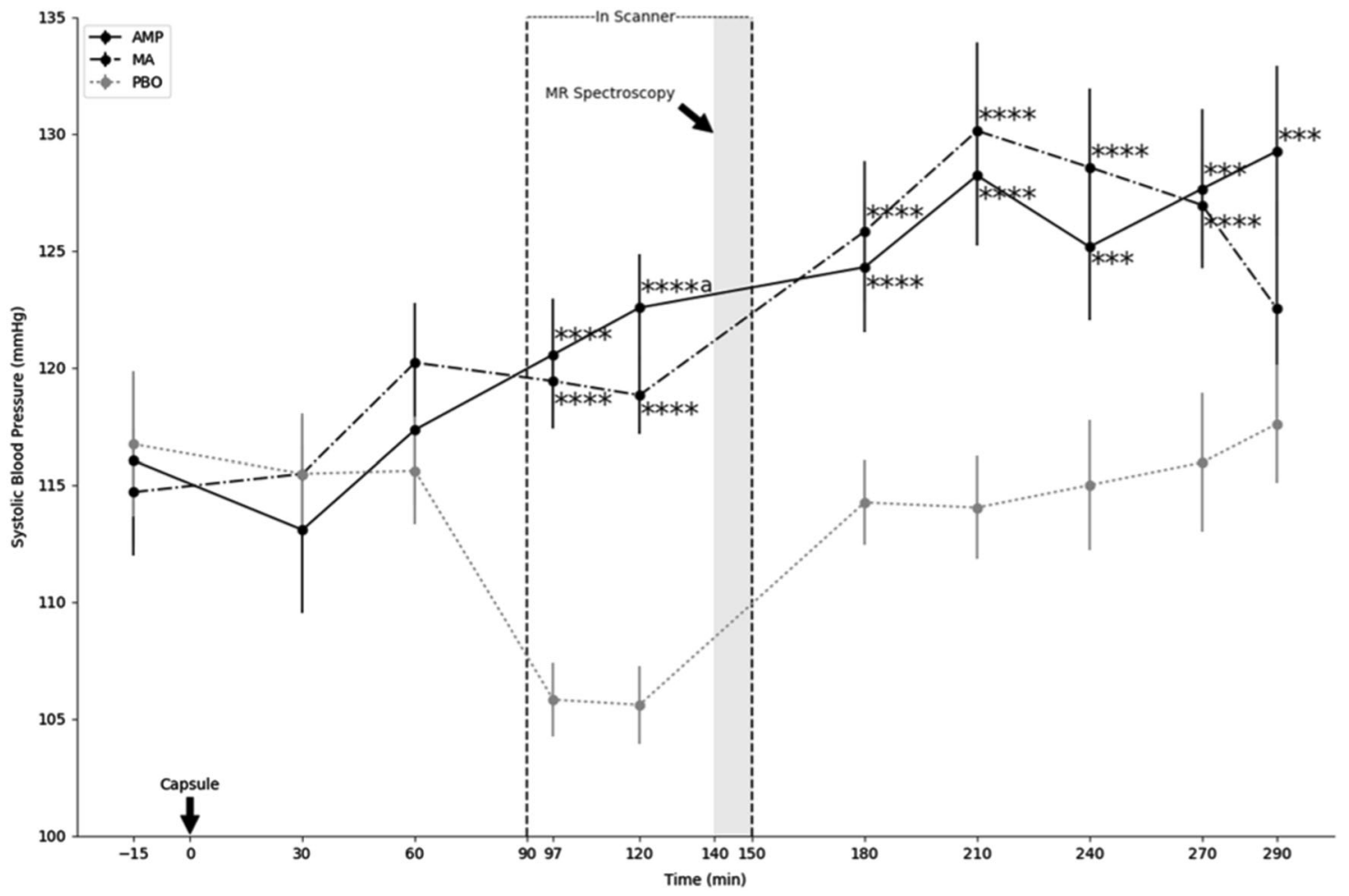

Fig. 3 Sympathomimetic activity of AMP and MA. X-axis indicates time elapsed from administration of the blinded study capsule. $Y$-axis indicates systolic blood pressure. Test sessions were $5.5 \mathrm{~h}$ in duration. MR spectroscopy was conducted 140-150 min after the capsule was administered (gray shading). Physiological monitoring was conducted at half hour intervals throughout the protocol. Time points of capsule administration, entry into the scanner, initiation of MR spectroscopy, and exit from scanner are indicated. AMP $d$-amphetamine (solid black line), MA methamphetamine (dashed black line), PBO placebo (dotted gray line). Error bars represent $\pm 1 \mathrm{SE}$. ${ }^{* * *} p \leq .001,{ }^{* * *} p \leq .005$ compared to PBO; ${ }^{a} \mathrm{AMP}$ and MA differ at $p<.05 . n=23$ with full data for analysis, see Suppl. Methods for details

\begin{tabular}{|c|c|c|c|c|c|c|}
\hline & \multicolumn{2}{|l|}{ PBO } & \multicolumn{2}{|l|}{ AMP } & \multicolumn{2}{|l|}{ MA } \\
\hline & Mean & SD & Mean & SD & Mean & SD \\
\hline \multicolumn{7}{|c|}{ Primary measures } \\
\hline Glx & 12.92 & 1.44 & $13.91 * * * a$ & 1.51 & 13.16 & 1.49 \\
\hline tNAA & 8.18 & 0.83 & 8.32 & 0.71 & 8.20 & 0.65 \\
\hline $\mathrm{tCr}$ & 6.28 & 0.54 & $6.56^{* * a}$ & 0.64 & 6.34 & 0.53 \\
\hline Cho & 1.80 & 0.19 & $1.91^{*}$ & 0.19 & 1.82 & 0.20 \\
\hline Ins & 4.80 & 0.50 & $5.12^{*}$ & 0.55 & 4.86 & 0.58 \\
\hline \multicolumn{7}{|c|}{ Subcomponents of $G / x$} \\
\hline Glu & 8.62 & 0.84 & $9.32^{* * * * a}$ & 1.01 & 8.80 & 1.08 \\
\hline Gln & 4.41 & 0.87 & 4.59 & 0.85 & 4.42 & 0.95 \\
\hline \multicolumn{7}{|c|}{$\begin{array}{l}\text { CSF-corrected metabolite levels in institutional units (i.u.) PBO placebo, } \\
A M P d \text {-amphetamine, } M A \text { methamphetamine, GIx Glx complex, } t N A A \text { total } \\
N \text {-acetyl-aspartate and } N \text {-acetylaspartyl-glutamic acid, } t C r \text { total creatine and } \\
\text { phosphocreatine, Cho choline-containing compounds, Ins myo-inositol, } \\
\text { Glu glutamate, Gln glutamine }{ }^{*} \text { Significantly different from placebo at }{ }^{*} p \\
<.05,{ }^{* *} p<.01,{ }^{* * *} p<.005,{ }^{* * * *} p<.0001^{\text {a }} \text { Survives Bonferroni correction for } \\
\text { multiple comparisons (adjusted alpha }=.007 \text { ). } n=24,63-66 \text { MRS spectra } \\
\text { per outcome }\end{array}$} \\
\hline
\end{tabular}

$=.18 ; \mathrm{CRLB}[\mathrm{GIn}]:$ Wald $\chi^{2}(2)=.425, p=.81 ;$ FWHM: Wald $\chi^{2}(2)$ $=.959, p=.62$; SNR: Wald $\left.\chi^{2}(2)=.774, p=.68\right)$. Voxel placement. Euclidian distances $(\mathrm{mm})$ between the center coordinates of the voxels were small (mean $\pm S D=3.4 \pm 2.0 \mathrm{~mm}$ ) with a minimum distance of $0.4 \mathrm{~mm}$. The center distances did not differ by drug condition ( $p=.32$, n.s.), indicating the reliability of voxel replacement across test sessions. Voxel composition. Segmentation procedures indicated a mean \pm SD tissue fraction of $.70 \pm .06$ gray matter, $.13 \pm .09$ white matter, and $.18 \pm .05$ CSF within the dACC voxel. Gray matter (Wald $X^{2}(2)=1.752, p=.42$ ), white matter (Wald $X^{2}(2)=3.254, p=.20$ ), and CSF fractions (Wald $\chi^{2}(2)=4.157$, $p=.13)$ did not differ between drug and PBO sessions. \%Voxel overlap. Sørensen-Dice coefficient (\%voxel overlap) was high (overall mean $\pm S D=64 \% \pm 12$ )) with a maximum of $85 \%$. Mean \pm SD Sørensen-Dice coefficient was $66 \% \pm 13$ for AMP and PBO, $63 \% \pm 14$ for MA and PBO, and $62 \% \pm 9$ for AMP and MA.

Sørensen-Dice coefficients did not differ between sessions $(p>.60$, n.s.). A representative image (within-subject Sørensen-Dice coefficient value of 65\%) is presented in Figure S2. Order. There was no effect of order of drug administration on any outcome (Glx: Wald $X^{2}(2)=.58, p=.75$, n.s.; Glu: Wald $X^{2}(2)=.97, p=.62, \mathrm{n}$. s.; tCr: Wald $X^{2}(2)=3.70, p=.16$, n.s.).

Relationship of Glx, Glu, and Gln

Glx was positively correlated with Glu and Gln on all three test sessions (Glu: $r>.73, p<.001$; Gln: $r>.74, p<.001$ ). In contrast, Glu and Gln were uncorrelated on all three sessions ( $\left.p^{\prime} s>.18\right)$. There was a $31 \%$ increase in the contribution of Glu to Glx under AMP compared to PBO conditions ( $71 \%$ shared variance during AMP; versus $54 \%$ shared variance during $\mathrm{PBO}$ ), consistent with the observed elevation in the level of Glu after AMP.

Mood correlates. AMP effects. AMP-induced change in $\mathrm{Glu}$ and $\mathrm{tCr}$ was positively correlated with the duration of participants' peak "Like Drug" ratings for AMP, $r=+.49, p=.02$, and $r=+.41, p$ $=.047$. These effects are illustrated in Fig. 6a, b. MA effects. MAinduced change in Glu and Glx was positively correlated with the magnitude of peak DEQ "Feel High" experienced after MA, $r=$ 
A

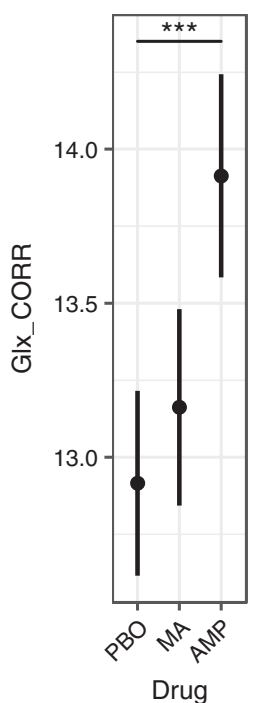

B

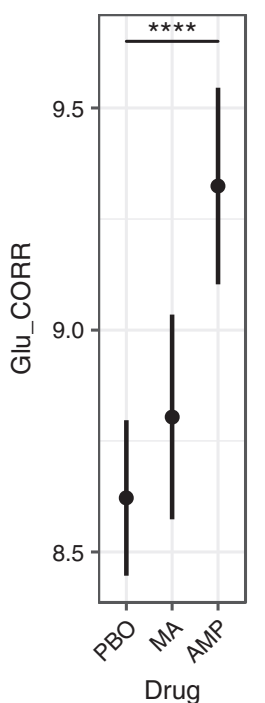

C

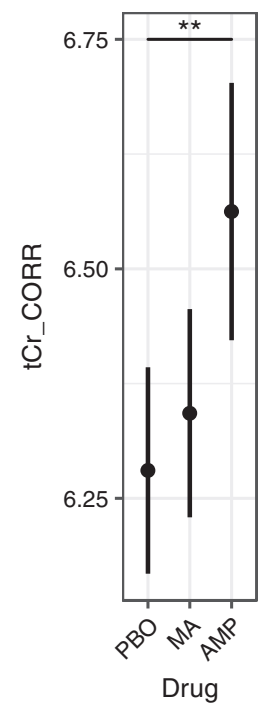

Fig. 4 Drug main effects. a Glx levels were higher during amphetamine sessions than placebo $\left.{ }^{* * *} p<.003\right)$, but not methamphetamine $(p=.078)$. b Similarly, Glu levels were higher during amphetamine sessions than placebo $\left({ }^{* * * *} p=.0001\right)$, but not methamphetamine $(p=.060)$. c tCr levels were higher during amphetamine sessions than placebo $(* * p=.0067)$, but not methamphetamine $(p=.067)$. Glx, Glu and $\mathrm{tCr}$ during methamphetamine and placebo did not differ significantly $(p>.470)$. Data are presented as mean \pm SEM. $Y$ axes are in institutional units (i.u.). Glx combined measure of glutamate and glutamine, Glu glutamate, $\mathrm{tCr}$ combined measure of creatine and phosphocreatine, PBO placebo, MA methamphetamine, AMP $d$-amphetamine. All metabolites were corrected for partial volume effects. $Y$ axes indicate CSF-corrected metabolite levels in institutional units (i.u.). $n=24$ participants with 66 scans for analysis (see Materials and methods section for details)

$+.52, p=.016$, and $r=+.42, p=.049$. These effects are illustrated in Fig. $6 \mathrm{c}, \mathrm{d}$. Effect magnitude. Drug-induced rise in Glu accounted for $24 \%$ of between-person variation in the duration of the "Like Drug" response to AMP, and $27 \%$ of between-person variation in the magnitude of the "Feel High" response to MA, with $38-42 \%$ of the predictable variance explained (see Suppl. Methods for details). Drug-induced changes in metabolites were strongly positively associated with the duration of AMP liking and the magnitude of the MA drug high reported by participants during the test sessions.

\section{DISCUSSION}

There were five main findings. First, AMP increased the level of Glx and Glu in the dACC. Second, there was high concordance between AMP effects on Glx and Glu. Third, we observed significant gender-related heterogeneity in participants' Glu response to MA. Lastly, there was a positive association between AMP effects on Glu and $\mathrm{tCr}$ and the duration of drug liking response to $A M P$, and a positive association between MA effects on Glu and Glx and peak ratings of drug high to MA.

Interpretation

Given the size of the MRS voxel $\left(10 \times 10 \times 15 \mathrm{~mm}^{3} ; 2250 \mathrm{~mm}^{3}\right)$, it is unlikely that alterations in glutamate release, reuptake, or transport could explain the present findings, as any such changes occur intra-synaptically and are detected on a spatial scale of 20-40 nm. Changes in extracellular Glu thus could not account for the increase in the level of glutamate we observe in the present MRS voxel, which identified drug-induced changes in Glu over a
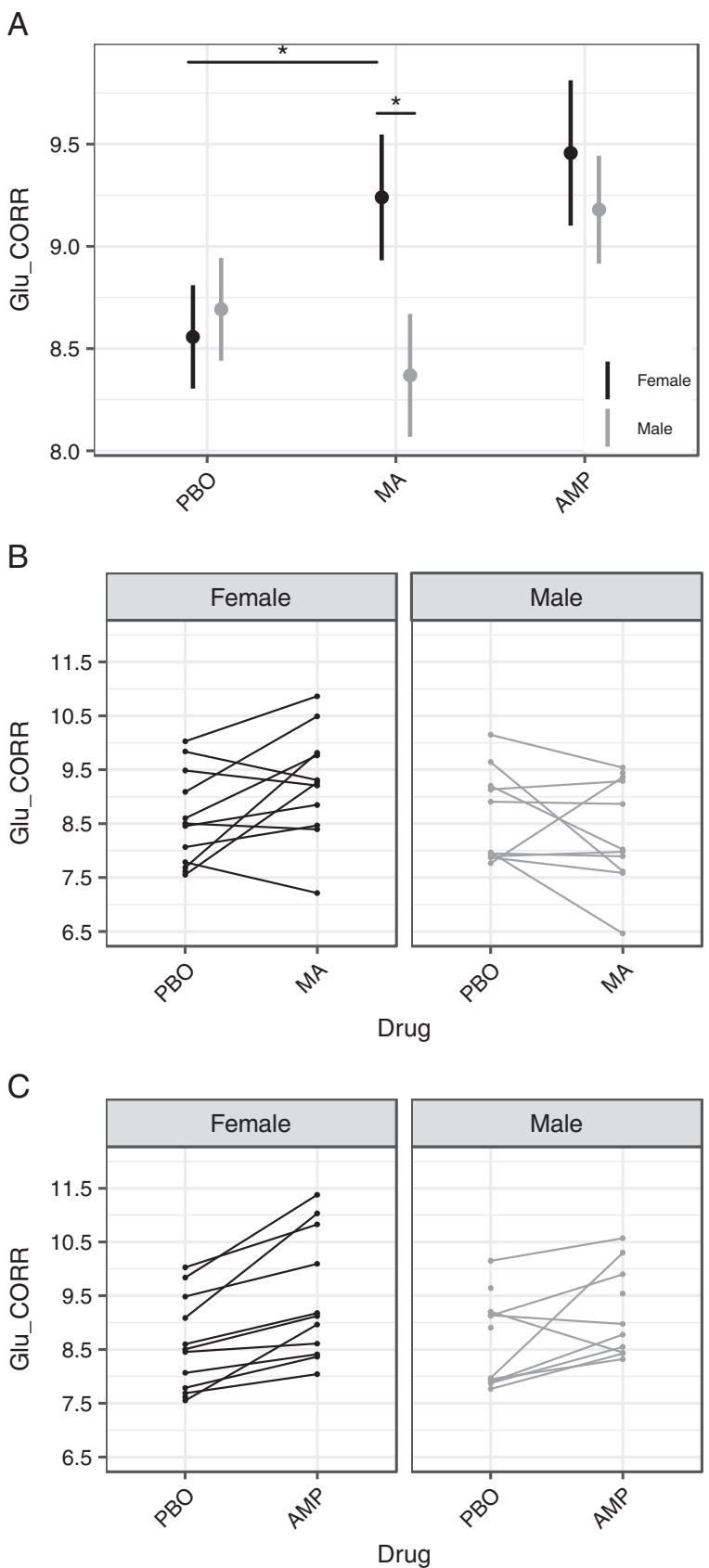

Fig. 5 Subject-level heterogeneity in Glu. a Gender-related heterogeneity in Glu. MA effects on Glu showed a significant drug $\times$ gender interaction, $B(S E)=-.99(.40), p=.020$. Pairwise test on MA and $\mathrm{PBO}$ conditions in females was significant, $t(21.97)=2.333, p$ $=.029$. In the MA condition Glu was significantly higher in women compared to men, $t(39.31)=-2.162, p=.037$. Error bars represent \pm $1 \mathrm{SE} .{ }^{*} p<.05$. b, c Subject-level data for Glu response to MA and AMP, coded by gender (black and gray lines denote females and males, respectively). Unconnected points indicate partial data (see Materials and methods section for details). All metabolites were corrected for partial volume effects. $Y$ axes indicate CSF-corrected metabolite levels in institutional units (i.u.). $n=24$ participants with 66 scans for analysis (see Materials and methods). Glu glutamate, PBO placebo, MA methamphetamine, AMP $d$-amphetamine

much larger spatial scale $\left(2250 \mathrm{~mm}^{3}\right)$. Moreover, as nearly the entire MRS-visible Glu signal is intracellular $(1000 \times$ higher concentration intra vs. extracellular), it is highly unlikely that the observed drug effects constitute an effect on extracellular Glu. We 
A

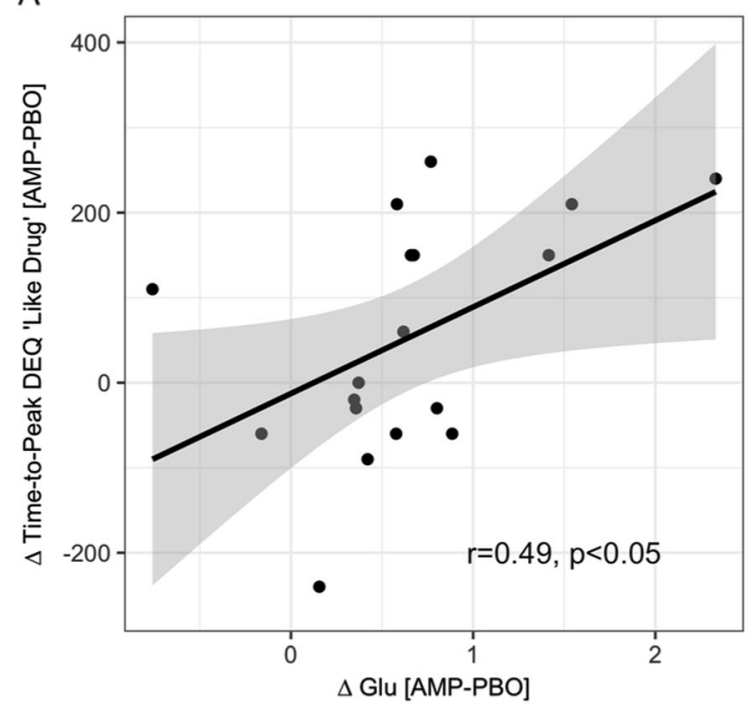

C

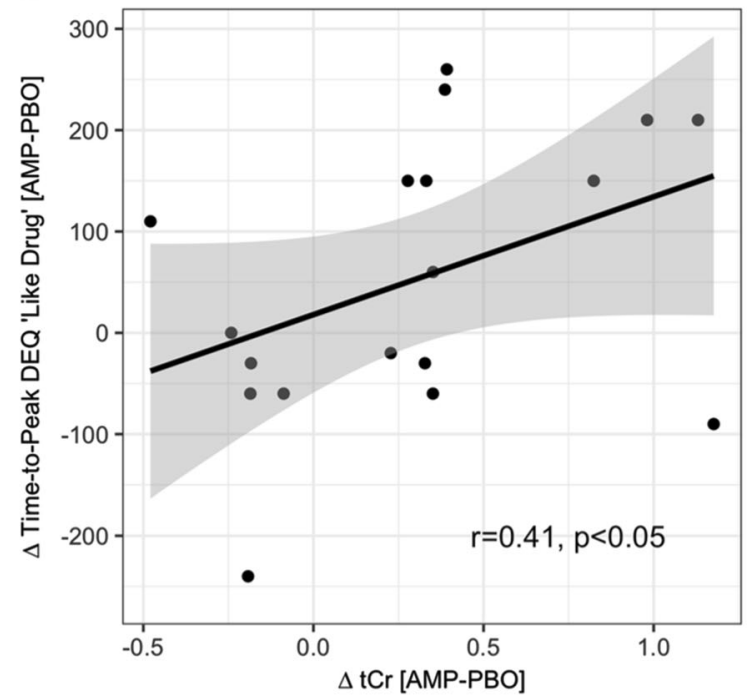

B

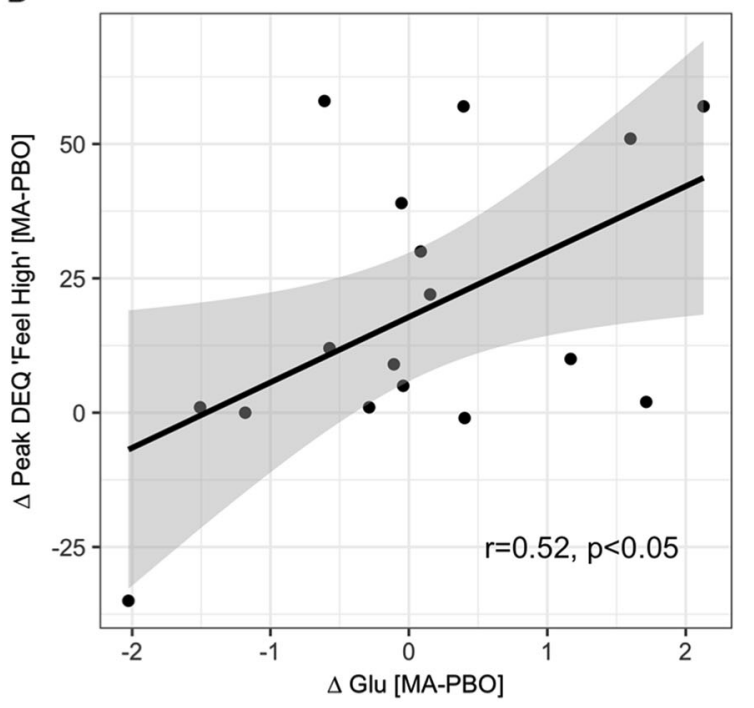

D

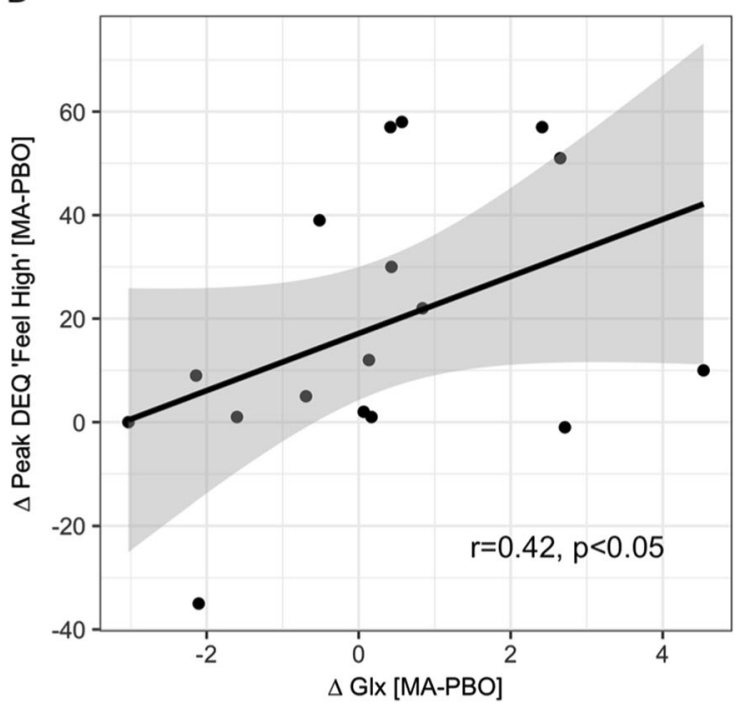

Fig. 6 Mood correlates. a AMP change in Glu ( $\Delta$ Glu) is positively correlated with the duration of peak DEQ "Like Drug" responses to AMP ( $\Delta$ Time-to-Peak DEQ "Like Drug"), $r=+.49, p=.02$. b AMP change in $\mathrm{tCr}(\Delta \mathrm{tCr})$ is positively correlated with the duration of peak DEQ "Like Drug" responses to AMP ( $\Delta$ Time-to-Peak DEQ "Like Drug"), $r=+.41, p=.047$. c MA change in Glu ( $\Delta$ Glu) is positively correlated with peak DEQ "Feel High" responses to MA ( $\Delta$ Peak DEQ "Feel High"), $r=+.52, p=.016$. d MA change in Glx $(\Delta$ Glx) is positively correlated with peak DEQ "Feel High" responses to MA ( $\Delta$ Peak DEQ "Feel High"), $r=+.42, p=.049$. Glx glutamate, tCr total creatine, Glx glutamate and glutamine. All metabolites were corrected for partial volume effects, see Materials and methods. Details on $\Delta$ Glu, Glx, tCr, DEQ "Like Drug," and DEQ "Feel High" ratings are in Suppl. Methods. Gray shading is $95 \%$ confidence interval. $n=17-18$ participants with full data for analysis, see Suppl. Methods for details

thus interpret the data as indicating a within-subject change in the overall concentration of intracellular glutamate in the dACC.

\section{Mechanisms}

Several mechanisms could underlie the drug-induced rise in Glu we observe in the dACC: an increased rate of Glu synthesis, increased availability of Glu precursors, and/or reductions in Glu catabolism within the voxel under drug. These mechanisms are not mutually exclusive. Drug effects on Glu synthesis. AMP has known effects on Glu synthesis through drug effects on glutaminase (GLS), which synthesizes Glu from intracellular Gln. In rodents, AMP potentiates GLS activity [31], and genetic knockdown of GLS1 mice display coordinated attenuations in GLS activity, frontal cortical Glu and behavioral responses to AMP [71]. The present data suggest that this enzymatic pathway may not be involved, however, as Gln levels were not observed to decrease (see Table 2); and as $\sim 80 \%$ of $\mathrm{Gln}$ in the brain is dedicated to GluGln cycling, see ref. [72]. It is more likely that AMP and MA affect Glu formation through the glycogenolysis pathway and TCA cycle $[73,74]$. Drug effects on Glu precursor availability. The impact of AMP and MA on heart rate and blood pressure (Table 1 and Fig. 3 ) contributes to an increased supply of glucose to the dACC, thereby facilitating the net creation of new Glu molecules within the voxel through an increase in Glu precursor availability. Glucose is the principle source of carbon skeletons needed to synthesize new Glu molecules under aerobic conditions, with the glycogenolysis pathway catalyzing the conversion of glucose to Glu via breakdown of glycogen [74]. As noradrenergic agonists facilitate glycogenolysis [73], the well-established noradrenergic effects of AMP and MA would further potentiate 
the formation of Glu through this pathway. Drug effects on Glu catabolism. Stimulant drugs also have dose- and region-specific effects on Glu catabolism. In rodents, shorter regimens and lower doses of AMP reduce limbic expression of glutamate decarboxylase (GAD), which catalyzes Glu conversion to GABA [75] and longer regimens and higher doses of AMP foster GAD expression [76]. A single dose of AMP in rodents also alters GAD mRNA within $30 \mathrm{~min}$ to $2 \mathrm{~h}$ of acute drug exposure $[30,75]$. Other catabolic enzymes affected by psychostimulant drugs include glutamine synthase, which converts Glu into Gln, and glutamate dehydrogenase, which metabolizes Glu into a-ketoglutarate [77-79]. A slowing of any of these catabolic processes would increase the level of intracellular Glu within the voxel. Summary of mechanisms. Based on this literature, the spatial resolution of our MRS voxel, the temporal features of our drug administration procedure, and the established sympathomimetic action of AMP and MA on heart rate and blood pressure, the present observations of increased Glu under drug are most consistent with an underlying effect of AMP on increased creation of new Glu molecules in the voxel, increased availability of Glu precursors, and reduced Glu catabolism. These three mechanisms likely account for the net increase in Glu we observe under drug.

\section{Mood correlates}

We found that drug-induced changes in dACC Glu correlated with the extent and duration of drug liking and drug high. Correlations were on the order of .4-.5, and explained $17-27 \%$ of the total variance. Given that the maximal correlation between imagingbased measures and self-report measures is constrained by the extent of test-retest instability in both sets of measures [80], drug changes in Glu likely account for $\sim 38-42 \%$ of the predictablevariance in drug liking and drug high. This is a substantive finding, and may be mechanistically important. Drug liking for psychostimulants is known to be immune to DA antagonism, as drug liking and drug high ratings to psychostimulants survive coadministration of DA antagonists [48, 81-83]. The present findings indicate a substantive role for Glu in the extent and duration of drug liking and drug high responses to psychostimulants in healthy young adults.

\section{Implications}

The well-established role of Glu in learning indicates the potential functional importance of the increased Glu observed here after AMP consumption. Increased levels of Glu within the dACC could alter learning and memory processes including state-dependent learning and cue conditioning. AMP-induced Glu could also affect subsequent drug choice behavior by facilitating underlying processes of operant learning, associative learning, and behavioral sensitization [84, 85]. Our findings suggest that such Gludependent processes may be altered following AMP consumption in healthy young adults.

The between-subject heterogeneity we observed in the glutamatergic response to MA (Fig. 5) is consistent with a heightened vulnerability to psychostimulants in females. In rodents, psychostimulants produce greater behavioral sensitization and dopamine release in females than males [86, 87]. In humans, female drug users report greater MA-induced euphoria, more often identify MA as their drug of choice and develop greater dependence on MA compared to males [88-90].

\section{Strengths and limitations}

The strengths of the study include the placebo-controlled, withinsubjects crossover design; evaluation of two prescription psychostimulants ( $d$-amphetamine and methamphetamine) in the same participants; time-locked procedures for drug administration; and a moderately sized sample of healthy volunteers carefully screened for medical and psychiatric disease. The sample was precisely balanced for gender and was racially and ethnically diverse, increasing generalizability. The within-subjects, placebocontrolled crossover design allows for strong inferences about drug effects on metabolites. There were multiple controls for MRS data quality and metabolites were evaluated using a wellestablished analytic strategy. Voxel placement was performed by an operator who was blinded to the drug condition and drug condition was randomized to eliminate bias in voxel placement based on drug condition. Achieved voxel overlap (64\%) did not differ between conditions and is on the order of that achieved by automated voxel placement [91], indicating excellent and stable within-subject reliability of voxel placement. Physiological data confirmed sympathomimetic drug activity, providing evidence of internal validity. Drug effects on Glx and Glu were positively related with drug-induced changes in mood, predicting duration of AMP liking and magnitude of drug high to MA. As these effects were observed concurrently (i.e., in the $5.5 \mathrm{~h}$ following acute exposure; see Fig. 1), these findings suggest a causal relationship between drug-induced changes in Glu and drug-induced changes in mood. These findings represent the first evidence of a relationship between acute changes in Glu and positively valenced emotion in humans. The within-subjects design provided three times the number of data points per subject compared to a between-subjects design and entirely controlled for individual differences among the drug conditions. This design substantially reduces error variance and maximizes statistical power compared to alternative designs.

Limitations of the design should also be noted. ${ }^{1} \mathrm{H}$ MRS was conducted solely in the dACC, thus the study does not provide information on other brain regions. Further, potential effects outside of the 140-150 min post-drug window would be missed. Use of a standard MRS PRESS acquisition precluded investigation of several metabolites including GABA and glutathione, which require spectral editing. As MRS provides information on total tissue metabolite in the voxel, Glx reflects both neurotransmitter and metabolic pools of Glu and Gln. Moreover, since Gln can be difficult to differentiate from Glu at $3 T$ and the chosen TE, additional studies using acquisition parameters that distinguish Gln from Glu are warranted. Future work using larger sample sizes, with oversampling of subjective states during the peak period of the drug response curve is recommended to improve temporal resolution and statistical power. Despite these caveats, the present findings provide the first evidence in humans that drug-induced changes in Glu correlate with subjective experiences of drug liking and drug high following drug ingestion.

\section{Conclusions and future directions}

In summary, we found that an acute single dose of AMP increased the concentration of Glu, Glx, and $\mathrm{tCr}$ in the dACC of healthy young adult volunteers. The increase in Glx is best explained by a drug-induced increase in intracellular Glu, as there was high concordance between AMP effects on Glx and our measure of Glu. Between-person heterogeneity in Glx and Glu responses to MA were significantly linked to gender. These findings are consistent with psychostimulant drug effects on Glu synthesis and catabolism in cell culture and in animals. AMP- and MA-induced changes in dACC Glu correlated with the duration and extent of drug liking and drug high, indicating a substantive association between glutamatergic responses and subjective experience after drug exposure. Drug-induced alteration in Glu levels within the dACC could affect myriad cognitive and behavioral processes, such as drug seeking, drug sensitization, state-dependent learning, memory, and cue conditioning $[84,85]$. To our knowledge, these findings provide the first evidence that specific psychostimulant drugs acutely alter the concentration of Glx and Glu in the human brain, and that psychostimulant-induced changes in Glu, Glx, and $\mathrm{tCr}$ predict acute changes in positively valenced emotion. Given the ability of psychostimulants to produce global changes in sympathomimetic activity and subjective emotion, drug effects on 
Glu likely transcend the present region (dACC). Future studies that apply whole-brain methods (i.e., 3D MRSI) will provide further regional information on stimulant drug effects on glutamatergic compounds in human neocortex.

\section{ACKNOWLEDGMENTS}

We thank Lynn Fanella, Erica Nixon, and Marilena DeMayo of the Brown University Magnetic Resonance Facility for technical assistance; Tim Souza for assistance with data processing; and Mikalei Gordon, Selen Senocak, and Nikitha Gangasani for assistance in ${ }^{1} \mathrm{H}$ MRS data collection. The authors are entirely responsible for the scientific content of the paper.

\section{FUNDING}

This work was supported by the National Institute of Health Grant DA029189 (T.L.W.); National Science Foundation Graduate Research Fellowship DGE1058262 (A.Z.N.); UTRA undergraduate teaching and research award from Brown University (C.A.B., T.L. W.); National Institute of Health Training Grant AA007459 and career award K23AA024704 (M.A.M.); National Institute of General Medical Sciences, National Institutes of Health (P20GM103645); National Institute of Health career award K01-AG050707 (A.J.W.); the National Center for Advancing Translational Sciences of the National Institutes of Health under University of Florida Clinical and Translational Science Awards KL2TR001429 and UL1TR001427; and National Institute of Health Grant AA007459. The views expressed in this article are those of the authors and do not necessarily reflect the position or policy of the Department of Veterans Affairs, United States government, National Institute of Health or National Science Foundation.

\section{AUTHOR CONTRIBUTIONS}

T.L.W. conceived the basic idea, initiated, designed, and directed the study and wrote the manuscript. T.L.W., C.A.B., A.Z.N., and E.G.W. designed procedures for voxel placement. C.A.B. and A.Z.N. acquired the majority of the MRS data. A.D.H. and E.C.P. designed and conducted the segmentation analysis for neuroanatomical data sets. $E$. G.W., M.A.M., A.J.W., and E.C.P. conducted the data quality control procedures. E.G.W., M.A.M., D.G.L., and T.L.W. performed the statistical and data analyses. E.C.P., A.J.W., A. D.H., R.A.C., T.L.W., S.F., D.G.L., E.G.W., and M.A.M. provided input on data analysis and the interpretation of results. T.L.W., A.D.H., M.A.M., E.C.P., S.F., D.G.L., A.J.W., R.A.C., and E.G.W. revised the manuscript. All authors read and approved the final manuscript.

\section{ADDITIONAL INFORMATION}

Supplementary Information accompanies this paper at https://doi.org/10.1038/ s41386-018-0027-7.

Competing interests: The authors declare no competing interests.

Publisher's note: Springer Nature remains neutral with regard to jurisdictional claims in published maps and institutional affiliations.

\section{REFERENCES}

1. Weyandt LL, Marraccini ME, Gudmundsdottir BG, Zavras BM, Turcotte KD, Munro $B A$, et al. Misuse of prescription stimulants among college students: a review of the literature and implications for morphological and cognitive effects on brain functioning. Exp Clin Psychopharmacol. 2013;21:385-407.

2. Rothman RB, Baumann MH. Monoamine transporters and psychostimulant drugs. Eur J Pharmacol. 2003;479:23-40.

3. Sulzer D, Sonders MS, Poulsen NW, Galli A. Mechanisms of neurotransmitter release by amphetamines: a review. Progress Neurobiol. 2005;75:406-33.

4. Carver CS, Johnson SL, Joormann J. Two-mode models of self-regulation as a tool for conceptualizing effects of the serotonin system in normal behavior and diverse disorders. Curr Dir Psychol Sci. 2009;18:195-9.

5. Coccaro EF, Fanning JR, Phan KL, Lee R. Serotonin and impulsive aggression. CNS Spectr. 2015;20:295-302.

6. Depue RA, White TL. Personality: neurobehavioral foundation and pharmacological protocols. In: Stolerman IP, Price LH, editors. Encyclopedia of Psychopharmacology. 2nd edn Heidelberg: Springer; 2015. p. 1258-66.

7. Goschke T, Bolte A. Emotional modulation of control dilemmas: the role of positive affect, reward, and dopamine in cognitive stability and flexibility. Neuropsychologia. 2014;62:403-23.

8. Perrier JF. Modulation of motoneuron activity by serotonin. Dan Med J. 2016;63:2.
9. Ramos BP, Arnsten AF. Adrenergic pharmacology and cognition: focus on the prefrontal cortex. Pharmacol Ther. 2007;113:523-36.

10. Flood JF, Baker ML, Davis JL. Modulation of memory processing by glutamic acid receptor agonists and antagonists. Brain Res. 1990;521:197-202.

11. Packard MG. Glutamate infused posttraining into the hippocampus or caudateputamen differentially strengthens place and response learning. Proc Natl Acad Sci USA. 1999;96:12881-6.

12. Quartermain D, Mower J, Rafferty MF, Herting RL, Lanthorn TH. Acute but not chronic activation of the NMDA-coupled glycine receptor with D-cycloserine facilitates learning and retention. Eur J Pharmacol. 1994;257:7-12.

13. Ernst T, Chang L. Adaptation of brain glutamate plus glutamine during abstinence from chronic methamphetamine use. J Neuroimmune Pharmacol. 2008;3:165-72.

14. Ernst T, Chang L, Leonido-Yee M, Speck O. Evidence for long-term neurotoxicity associated with methamphetamine abuse: a 1H MRS study. Neurology. 2000;54:1344-9.

15. Nordahl TE, Salo $R$, Natsuaki $Y$, Galloway GP, Waters $C$, Moore $C D$, et al. Methamphetamine users in sustained abstinence: a proton magnetic resonance spectroscopy study. Arch Gen Psychiatry. 2005;62:444-52.

16. Nordahl TE, Salo R, Possin K, Gibson DR, Flynn N, Leamon M, et al. Low N-acetylaspartate and high choline in the anterior cingulum of recently abstinent methamphetamine-dependent subjects: a preliminary proton MRS study. Magnetic resonance spectroscopy. Psychiatry Res. 2002;116:43-52.

17. Salo $R$, Nordahl TE, Natsuaki $Y$, Leamon MH, Galloway GP, Waters $C$, et al. Attentional control and brain metabolite levels in methamphetamine abusers. Biol Psychiatry. 2007;61:1272-80.

18. Schmaal L, Veltman DJ, Nederveen A, van den Brink W, Goudriaan AE. Nacetylcysteine normalizes glutamate levels in cocaine-dependent patients: a randomized crossover magnetic resonance spectroscopy study. Neuropsychopharmacology. 2012;37:2143-52.

19. Sekine $Y$, Minabe $Y$, Kawai $M$, Suzuki $K$, lyo $M$, Isoda $H$, et al. Metabolite alterations in basal ganglia associated with methamphetamine-related psychiatric symptoms. A proton MRS study. Neuropsychopharmacology. 2002;27:453-61.

20. Sung YH, Cho SC, Hwang J, Kim SJ, Kim H, Bae S, et al. Relationship between Nacetyl-aspartate in gray and white matter of abstinent methamphetamine abusers and their history of drug abuse: a proton magnetic resonance spectroscopy study. Drug Alcohol Depend. 2007;88:28-35.

21. Sung YH, Yurgelun-Todd DA, Shi XF, Kondo DG, Lundberg KJ, McGlade EC, et al. Decreased frontal lobe phosphocreatine levels in methamphetamine users. Drug Alcohol Depend. 2013;129:102-9.

22. Rae CD. A guide to the metabolic pathways and function of metabolites observed in human brain 1H magnetic resonance spectra. Neurochem Res. 2014;39:1-36.

23. Goryawala MZ, Sheriff $S$, Maudsley AA. Regional distributions of brain glutamate and glutamine in normal subjects. NMR Biomed. 2016;29:1108-16.

24. Zwanzger P, Zavorotnyy M, Gencheva E, Diemer J, Kugel H, Heindel W, et al. Acute shift in glutamate concentrations following experimentally induced panic with cholecystokinin tetrapeptide--a 3T-MRS study in healthy subjects. Neuropsychopharmacology. 2013;38:1648-54.

25. Rowland LM, Bustillo JR, Mullins PG, Jung RE, Lenroot R, Landgraf E, et al. Effects of ketamine on anterior cingulate glutamate metabolism in healthy humans: a 4T proton MRS study. Am J Psychiatry. 2005;162:394-6.

26. Zhang H, Wang W, Gao W, Ge Y, Zhang J, Wu S, et al. Effect of propofol on the levels of neurotransmitters in normal human brain: a magnetic resonance spectroscopy study. Neurosci Lett. 2009;467:247-51.

27. Albrecht J, Zielinska M. Mechanisms of excessive extracellular glutamate accumulation in temporal lobe epilepsy. Neurochem Res. 2016;42:1724-34.

28. Hunter MA, Coffman BA, Gasparovic C, Calhoun VD, Trumbo MC, Clark VP. Baseline effects of transcranial direct current stimulation on glutamatergic neurotransmission and large-scale network connectivity. Brain Res. 2015;1594:92-107.

29. Kalivas PW. Cocaine and amphetamine-like psychostimulants: neurocircuitry and glutamate neuroplasticity. Dialog Clin Neurosci. 2007;9:389-97.

30. Lai CC, Lee LJ, Yin HS. Combinational effects of ketamine and amphetamine on behaviors and neurotransmitter systems of mice. Neurotoxicology. 2013;37:136-43.

31. Sherman AD, Mott J. Amphetamine stimulation of glutaminase is blocked by neuroleptics. Life Sci. 1985;36:1163-7.

32. Underhill SM, Wheeler DS, Li M, Watts SD, Ingram SL, Amara SG. Amphetamine modulates excitatory neurotransmission through endocytosis of the glutamate transporter EAAT3 in dopamine neurons. Neuron. 2014;83:404-16.

33. de Bruijn ER, Hulstijn W, Verkes RJ, Ruigt GS, Sabbe BG. Drug-induced stimulation and suppression of action monitoring in healthy volunteers. Psychopharmacology. 2004;177:151-60.

34. Barrett LF, Satpute AB. Large-scale brain networks in affective and social neuroscience: towards an integrative functional architecture of the brain. Curr Opin Neurobiol. 2013;23:361-72. 
35. Vollm BA, de Araujo IE, Cowen PJ, Rolls ET, Kringelbach ML, Smith KA, et al. Methamphetamine activates reward circuitry in drug naive human subjects. Neuropsychopharmacology. 2004;29:1715-22.

36. Del Arco A, Gonzalez-Mora JL, Armas VR, Mora F. Amphetamine increases the extracellular concentration of glutamate in striatum of the awake rat: involvement of high affinity transporter mechanisms. Neuropharmacology. 1999;38:943-54.

37. Del Arco A, Martinez R, Mora F. Amphetamine increases extracellular concentrations of glutamate in the prefrontal cortex of the awake rat: a microdialysis study. Neurochem Res. 1998;23:1153-8.

38. Harvey BH, Brink CB, Seedat S, Stein DJ. Defining the neuromolecular action of myo-inositol: application to obsessive-compulsive disorder. Prog Neuropsychopharmacol Biol Psychiatry.2002;26:21-32.

39. Silverstone PH, Rotzinger S, Pukhovsky A, Hanstock CC. Effects of lithium and amphetamine on inositol metabolism in the human brain as measured by $1 \mathrm{H}$ and 31P MRS. Biol Psychiatry. 1999;46:1634-41.

40. White TL, Lejuez CW, de Wit H. Personality and gender differences in effects of damphetamine on risk taking. Exp Clin Psychopharmacol. 2007;15:599-609.

41. White TL, Lott DC, de Wit H. Personality and the subjective effects of acute amphetamine in healthy volunteers. Neuropsychopharmacology. 2006;31:1064-74.

42. Kahlig KM, Binda F, Khoshbouei H, Blakely RD, McMahon DG, Javitch JA, et al. Amphetamine induces dopamine efflux through a dopamine transporter channel. Proc Natl Acad Sci USA. 2005;102:3495-3500.

43. Pifl $\mathrm{C}$, Agneter $\mathrm{E}$, Drobny $\mathrm{H}$, Sitte $\mathrm{HH}$, Singer EA. Amphetamine reverses or blocks the operation of the human noradrenaline transporter depending on its concentration: superfusion studies on transfected cells. Neuropharmacology. 1999:38:157-65.

44. Martin WR, Sloan JW, Sapira JD, Jasinski DR. Physiologic, subjective, and behavioral effects of amphetamine, methamphetamine, ephedrine, phenmetrazine, and methylphenidate in man. Clin Pharmacol Ther. 1971;12:245-58.

45. Mayo LM, de Wit $\mathrm{H}$. Acquisition of responses to a methamphetamine-associated cue in healthy humans: self-report, behavioral, and psychophysiological measures. Neuropsychopharmacology. 2015;40:1734-41.

46. Lamb RJ, Henningfield JE. Human d-amphetamine drug discrimination: methamphetamine and hydromorphone. J Exp Anal Behav. 1994;61:169-80.

47. Wachtel SR, de Wit $H$. Subjective and behavioral effects of repeated $d$ amphetamine in humans. Behav Pharmacol. 1999;10:271-81.

48. Wachtel $\mathrm{SR}$, Ortengren $\mathrm{A}$, de Wit $\mathrm{H}$. The effects of acute haloperidol or risperidone on subjective responses to methamphetamine in healthy volunteers. Drug Alcohol Depend. 2002;68:23-33.

49. Edden RA, Puts NA, Harris AD, Barker PB, Evans CJ. Gannet: a batch-processing tool for the quantitative analysis of gamma-aminobutyric acid-edited MR spectroscopy spectra. J Magn Reson Imaging. 2014;40:1445-52.

50. Ashburner J, Friston KJ. Unified segmentation. Neuroimage. 2005;26:839-51.

51. Harris AD, Puts NA, Edden RA. Tissue correction for GABA-edited MRS: considerations of voxel composition, tissue segmentation, and tissue relaxations. J Magn Reson Imaging. 2015:42:1431-40.

52. Grayson DS, Ray S, Carpenter S, lyer S, Dias TG, Stevens C, et al. Structural and functional rich club organization of the brain in children and adults. PLoS ONE. 2014;9:e88297.

53. Traber F, Block W, Lamerichs R, Gieseke J, Schild HH. 1H metabolite relaxation times at 3.0 tesla: measurements of $\mathrm{T} 1$ and $\mathrm{T} 2$ values in normal brain and determination of regional differences in transverse relaxation. J Magn Reson Imaging. 2004;19:537-45.

54. Provencher SW. Estimation of metabolite concentrations from localized in vivo proton NMR spectra. Magn Reson Med. 1993;30:672-9.

55. Ethofer T, Mader I, Seeger U, Helms G, Erb M, Grodd W, et al. Comparison of longitudinal metabolite relaxation times in different regions of the human brain at 1.5 and 3 Tesla. Magn Reson Med. 2003;50:1296-301.

56. Mlynarik V, Gruber S, Moser E. Proton T (1) and T (2) relaxation times of human brain metabolites at 3 Tesla. NMR Biomed. 2001;14:325-31.

57. Geurts JJ, Barkhof F, Castelijns JA, Uitdehaag BM, Polman $\mathrm{CH}$, Pouwels PJ. Quantitative 1H-MRS of healthy human cortex, hippocampus, and thalamus: metabolite concentrations, quantification precision, and reproducibility. J Magn Reson Imaging. 2004;20:366-71.

58. Baker EH, Basso G, Barker PB, Smith MA, Bonekamp D, Horska A. Regional apparent metabolite concentrations in young adult brain measured by (1)H MR spectroscopy at 3 Tesla. J Magn Reson Imaging. 2008;27:489-99.

59. Zhu H, Barker PB. MR spectroscopy and spectroscopic imaging of the brain. Methods Mol Biol. 2011;711:203-26.

60. Haga KK, Khor YP, Farrall A, Wardlaw JM. A systematic review of brain metabolite changes, measured with $1 \mathrm{H}$ magnetic resonance spectroscopy, in healthy aging. Neurobiol Aging. 2009;30:353-63.

61. Li BS, Wang H, Gonen $\mathrm{O}$. Metabolite ratios to assumed stable creatine level may confound the quantification of proton brain MR spectroscopy. Magn Reson Imaging. 2003;21:923-8.
62. Larsen RJ, Newman M, Nikolaidis A. Reduction of variance in measurements of average metabolite concentration in anatomically-defined brain regions. J Magn Reson. 2016;272:73-81

63. Maltezos S, Horder J, Coghlan S, Skirrow C, O'Gorman R, Lavender TJ, et al. Glutamate/glutamine and neuronal integrity in adults with ADHD: a proton MRS study. Transl Psychiatry. 2014;4:e373.

64. Brandt AS, Unschuld PG, Pradhan S, Lim IA, Churchill G, Harris AD, et al. Agerelated changes in anterior cingulate cortex glutamate in schizophrenia: a (1)H MRS Study at 7 Tesla. Schizophr Res. 2016;172:101-5.

65. Dice LR. Measures of the amount of ecologic association between species. Ecology. 1945;26:297-302.

66. Sørensen T. A method of establishing groups of equal amplitude in plant sociology based on similarity of species content and its application to analyses of the vegetation on Danish commons. Viden Selsk Biol Skr. 1948;5:1-34.

67. Morean ME, de Wit H, King AC, Sofuoglu M, Rueger SY, O'Malley SS. The drug effects questionnaire: psychometric support across three drug types. Psychopharmacology. 2013;227:177-92.

68. Lakens D. Calculating and reporting effect sizes to facilitate cumulative science: a practical primer for t-tests and ANOVAs. Front Psychol. 2013;4:863.

69. Cohen J. Statistical power analysis for the behavioral sciences. 2nd ed. New Jersey: Lawrence Erlbaum Associates Inc; 1988.

70. Faul F, Erdfelder E, Buchner A, Lang AG. Statistical power analyses using G*Power 3.1: tests for correlation and regression analyses. Behav Res Methods. 2009;41:1149-60.

71. Gaisler-Salomon I, Miller GM, Chuhma N, Lee S, Zhang H, Ghoddoussi F, et al. Glutaminase-deficient mice display hippocampal hypoactivity, insensitivity to pro-psychotic drugs and potentiated latent inhibition: relevance to schizophrenia. Neuropsychopharmacology. 2009;34:2305-22.

72. Ramadan S, Lin A, Stanwell P. Glutamate and glutamine: a review of in vivo MRS in the human brain. NMR Biomed. 2013;26:1630-46.

73. Gibbs ME. Role of glycogenolysis in memory and learning: regulation by noradrenaline, serotonin and ATP. Front Integr Neurosci. 2015;9:70.

74. Hertz L, Chen Y. Additional mechanisms for brain activation failure due to reduced glucose metabolism-a commentary on Zilberter and Zilberter: The vicious circle of hypometabolism in neurodegenerative diseases. J Neurosci Res. (2017) doi: 10.1002/jnr.24192.

75. Carta AR, Moreno CC, Cadoni C, Tronci E, Di Chiara G. Long-term increase in GAD67 mRNA expression in the central amygdala of rats sensitized by drugs and stress. Eur J Neurosci. 2008;27:1220-30.

76. Yin HS, Tan HW. Effects of amphetamine on serotoninergic and GABAergic expression of developing brain. Neurotoxicology Teratol. 2007;29: 264-72.

77. Pereira FC, Cunha-Oliveira T, Viana SD, Travassos AS, Nunes S, Silva C, et al. Disruption of striatal glutamatergic/GABAergic homeostasis following acute methamphetamine in mice. Neurotoxicology Teratol. 2012;34:522-9.

78. Stadlin A, Lau JW, Szeto YK. A selective regional response of cultured astrocytes to methamphetamine. Ann N Y Acad Sci. 1998;844:108-21.

79. Vicente-Rodriguez M, Gramage E, Herradon G, Perez-Garcia C. Phosphoproteomic analysis of the striatum from pleiotrophin knockout and midkine knockout mice treated with cocaine reveals regulation of oxidative stress-related proteins potentially underlying cocaine-induced neurotoxicity and neurodegeneration. Toxicology. 2013;314:166-73.

80. Lieberman MD, Berkman ET, Wager TD. Correlations in social neuroscience aren't voodoo: Commentary on Vul et al. (2009). Perspect Psychol Sci. 2009;4:299-307.

81. Brauer $\mathrm{LH}$, de Wit $\mathrm{H}$. Subjective responses to $d$-amphetamine alone and after pimozide pretreatment in normal, healthy volunteers. Biol Psychiatry. 1996;39:26-32.

82. Brauer LH, De Wit H. High dose pimozide does not block amphetamineinduced euphoria in normal volunteers. Pharmacol Biochem Behav. 1997a;56: 265-72.

83. Brauer LH, Goudie AJ, de Wit H. Dopamine ligands and the stimulus effects of amphetamine: animal models versus human laboratory data. Psychopharmacology. 1997;130:2-13.

84. Costa G, Morelli M, Simola N. Involvement of glutamate NMDA receptors in the acute, long-term, and conditioned effects of amphetamine on rat $50 \mathrm{kHz}$ ultrasonic vocalizations. Int J Neuropsychopharmacol. 2015;18:pyv057.

85. Stewart J, Druhan JP. Development of both conditioning and sensitization of the behavioral activating effects of amphetamine is blocked by the noncompetitive NMDA receptor antagonist, MK-801. Psychopharmacology. 1993;110:125-32.

86. Becker JB, Molenda H, Hummer DL. Gender differences in the behavioral responses to cocaine and amphetamine. Implications for mechanisms mediating gender differences in drug abuse. Ann N Y Acad Sci. 2001;937: $172-87$ 
Psychostimulant Drugs Increase Glutamate in Human Neocortex...

TL White et al.

87. Mathews IZ, McCormick CM. Female and male rats in late adolescence differ from adults in amphetamine-induced locomotor activity, but not in conditioned place preference for amphetamine. Behav Pharmacol. 2007;18:641-50.

88. Cretzmeyer M, Sarrazin MV, Huber DL, Block RI, Hall JA. Treatment of methamphetamine abuse: research findings and clinical directions. J Subst Abus Treat. 2003;24:267-77.

89. Dluzen DE, Liu B. Gender differences in methamphetamine use and responses: a review. Gend Med. 2008;5:24-35.
90. Rawson RA, Gonzales R, Obert JL, McCann MJ, Brethen P. Methamphetamine use among treatment-seeking adolescents in Southern California: participant characteristics and treatment response. J Subst Abus Treat. 2005;29:67-74.

91. Lee H, Caparelli E, Li H, Mandal A, Smith SD, Zhang S, et al. Computerized MRS voxel registration and partial volume effects in single voxel 1H-MRS. Magn Reson Imaging. 2013;31:1197-205. 